\section{Exploring pictures by hand}

Recently, Magee and Kennedy ${ }^{1}$ have found that blindfolded sighted people can identify a raised-line drawing more readily when the index finger of one hand is guided along its outline than when the finger is allowed to trace the figure without guidance. This finding could evidently be used in perfecting methods of teaching the blind how to use and interpret raised-line drawings.

However, three difficulties render the conclusions of this study inapplicable to the teaching of blind persons. First, the blindfolded condition is in no sense equivalent to, or a model for, blindness. The study should have been conducted both with blind children and with recently blinded adults, who may differ from each other and from the sighted population in the extent of their firsthand experience with objects whose twodimensional representations they are asked to identify tactually.

Second, and much more important, a blind person does not perceive a raisedline drawing by tracing its outline with the tip of one index finger; instead, the drawing is first scanned by several fingers of both hands, and later traced in segments and in various directions by the index fingers of each hand. This method is analogous to visual inspection of pictures. If a raised-line illustration consists of more than just an outline, the single-finger tracing method would be inefficient, confusing and uninformative.

Finally, the results that Magee and Kennedy obtained probably depend on the size of the drawing. From their data I calculate that it took an observer about $30 \mathrm{~s}$ to be guided around the perimeter of a swan whose representation fits in a $10-$ $\mathrm{cm}$ square. I suspect that single-finger tracing without guidance is far more efficient on a small drawing than on one as large as that used by Magee and Kennedy. In fact, moderately small-scale drawings are probably easier to interpret than large-scale illustrations, as was clearly recognized by those who developed and perfected the Braille alphabet.

In conclusion, the work of Magee and Kennedy is one of many examples of the tendency for research on blindness to be conducted with little understanding of blindness itself. Despite this ignorance, conclusions from such studies have great impact on the education of the blind.

Department of Zoology,

Gebrat J. Vermeij

University of Maryland

College Park, Maryland 20742

1. Magee, L. A. \& Kennedy, J. M. Nature 283, 287 (1980)

MaGeE AND KenNedy RePLy--Vermeij's response to our report ${ }^{1}$ is concerned with the practical application of our findings to the use of raised-line drawings by the blind. This is a fruitful approach to research in this area. Yet, one should not confuse an empirical method used to test a theory with an extrapolation to the pedagogy of the blind.

We tested the generality of a well established claim in haptic form perception-namely that unaided exploration is superior to aided exploration. Contrary to popular opinion, we found that self-guided exploration of raised-line drawings is inferior to aided exploration. We then used this result to evaluate the relative contribution of cutaneous and kinaesthetic sensitivity to identification and found that kinaesthetic information is predominant.

Vermeij does not question these basic findings but does ask whether they are applicable to the blind. It should be noted that traditionally (see, for example, ref. 2) the blindfolded, not the blind, have been used as subjects in experiments on haptic form perception. To the extent that the blind and blindfolded have that same physiological mechanisms underlying the receptive functions of the hand, we believe our results to be generally applicable. Indeed, Kennedy and Fox ${ }^{3}$ have found very little difference among the blindfolded, adventitiously blind and congenitally blind in their ability to identify raisedline drawings.

Vermeij says and we agree-that a blind person generally uses more than one finger when exploring a raised-line drawing. However, the crucial point is that a technique focusing on a single finger allows one to manipulate exploration and make comparisons in performance which would be exceedingly difficult, if not impossible, if 10 fingers had to be controlled simultaneously. Moreover, the blind are able to identify drawings with a single finger. Note, we are not proposing that single-finger tracing is optimal or establishing the efficacy of this mode of exploration compared to other strategies.

Vermeij speculates that our results probably depend on the size and complexity of the drawing. Our pilot studies have not found any interactions of this type.

In sum, our major theoretical findings are quite clear. Guidance can be useful in haptic form perception and the source of information subserving identification of raised lines is predominantly kinaesthetic. The practical implications are that a teacher need not refrain from helping a blind student explore raisedline figures and that an emphasis on movement variables may be beneficial when displays of this type are made.

\section{LOCHLAN E. MAGEF}

JOHN M. KENNEDY

Division of Life Science,

Scarborough College, West Hill,

Ontario, Canada MIC 1 A4

\footnotetext{
1. Mayee, L. E. \& Kennedy, J. M. Nature 283, 287 (3980

2. Gibson, J. J. Psychol. Rev. 69, 477 (1962).

3. Kennedy, J. M. \& Fox, N. in The Arts und Cognition teds Pcrkins, D. \& Leondar, B.) (Johns Hopkins University
} Press, Baltimore, 1977). 